\title{
CONVERGENCE TO STABLE LAWS IN RELATIVE ENTROPY
}

\author{
S. G. BOBKOV ${ }^{1,4}$, G. P. CHISTYAKOV ${ }^{2,4}$, AND F. GÖTZE ${ }^{3,4}$
}

\begin{abstract}
Convergence to stable laws in relative entropy is established for sums of i.i.d. random variables.
\end{abstract}

\section{Introduction}

Given independent identically distributed random variables $\left(X_{n}\right)_{n \geq 1}$, consider the normalized sums

$$
Z_{n}=\frac{X_{1}+\cdots+X_{n}}{b_{n}}-a_{n}
$$

defined for given (non-random) $a_{n} \in \mathbf{R}$ and $b_{n}>0$. Assume that $Z_{n}$ converges weakly in distribution to a random variable $Z$ which has a non-degenerate stable law. In this paper, we would like to study whether or not this convergence holds in a stronger sense. This question has been studied and affirmatively solved in the literature, for example, using the total variation distance between the distributions of $Z_{n}$ and $Z$ (cf. [I-L]). Results in the central limit theorem suggest to consider for instance the stronger "entropic" distance, that is, the relative entropy, where, however, not so much is known. Similarly one might consider the convergence in terms of the closely related Fisher information (a question raised in $[\mathrm{J}]$, Ch.5). Convergence in such distances may be viewed as part of a theoretic-information approach to limit theorems, which has been initiated by Linnik [Li], who studied the behavior of the entropy of sums of regularized independent summands in the central limit theorem.

Given random variables $X$ and $Z$ with distributions $\mu$ and $\nu$, respectively, the relative entropy of $\mu$ with respect to $\nu$, sometimes called informational divergence or Kullback-Leibler distance, of $\mu$ and $\nu$, is defined by

$$
D(X \| Z)=D(\mu \| \nu)=\int \log \frac{d \mu}{d \nu} d \mu
$$

provided that $\mu$ is absolutely continuous with respect to $\nu$ (and otherwise $D(X \| Z)=+\infty$ ).

We consider the relative entropy with respect to so-called non-extremal stable laws (cf. relations (1.1) below and the definition before them). The aim of this note is to prove:

1991 Mathematics Subject Classification. Primary 60E.

Key words and phrases. Entropy, entropic distance, central limit theorem, stable laws.

1) School of Mathematics, University of Minnesota, USA; Email: bobkov@math.umn.edu.

2) Faculty of Mathematics, University of Bielefeld, Germany; Email: chistyak@math.uni-bielefeld.de.

3) Faculty of Mathematics, University of Bielefeld, Germany; Email: goetze@math.uni-bielefeld.de.

4) Research partially supported by NSF grant and SFB 701. 
Theorem 1.1. Assume that the sequence of normalized sums $Z_{n}$ defined above converges weakly to a random variable $Z$ with a non-extremal stable limit law. Then the relative entropy distances converge to zero, that is, $D\left(Z_{n} \| Z\right) \rightarrow 0$, as $n \rightarrow \infty$, if and only if $D\left(Z_{n} \| Z\right)<+\infty$, for some $n$.

In the sequel, we consider non-degenerate distributions, only.

If $X_{1}$ has a finite second moment, weak convergence $Z_{n} \Rightarrow Z$ holds with $a_{n}=\sqrt{n} \mathbf{E} X_{1}$, $b_{n}=\sqrt{n}$, and $Z$ being normal. In this case, Theorem 1.1 turns into the entropic central limit theorem by Barron [B]; cf. also [B-C-G] for refinements and a different approach. Thus, Theorem 1.1 may be viewed as an extension of Barron's result.

If $X_{1}$ has an infinite second moment, but still belongs to the domain of normal attraction, it follows that $D\left(Z_{n} \| Z\right)=+\infty$ for all $n$. Hence, in this special case there is no convergence in relative entropy.

In the remaining cases $Z$ has a stable distribution with some parameters $0<\alpha<2$ and $-1 \leq \beta \leq 1$. It then has a continuous density $\psi(x)$ with characteristic function $f(t)=\mathbf{E} e^{i t Z}$ described by the formula

$$
\log f(t)=\exp \left\{i a t-c|t|^{\alpha}(1+i \beta \operatorname{sign}(t) \omega(t, \alpha))\right\},
$$

where $a \in \mathbf{R}, c>0$, and $\omega(t, \alpha)=\tan \left(\frac{\pi \alpha}{2}\right)$ in case $\alpha \neq 1$, and $\omega(t, \alpha)=\frac{2}{\pi} \log |t|$ for $\alpha=1$. In particular, $|f(t)|=e^{-c|t|^{\alpha}}$.

Exact expressions for the characteristic function are, however, not sufficiently informative for establishing results like Theorem 1.1. In order to pass from weak convergence to a stronger convergence, we need more information about the stable densities $\psi$.

This information in turn will depend on the type of a given stable distribution. A stable distribution is called non-extremal, if it is normal or, if $0<\alpha<2$ and $-1<\beta<1$. In the latter case, the density $\psi$ of $Z$ is positive on the whole real line and satisfies asymptotic relations

$$
\psi(x) \sim c_{0}|x|^{-(1+\alpha)} \quad(x \rightarrow-\infty), \quad \psi(x) \sim c_{1} x^{-(1+\alpha)} \quad(x \rightarrow+\infty)
$$

with some constants $c_{0}, c_{1}>0$ (cf. [I-L], [Z]).

The behavior of $\psi$ in the extremal case (when $|\beta|=1$ ) is different. For example, when $0<\alpha<1$, the density is positive on a half-axis $H=\left(x_{0},+\infty\right)$ or $H=\left(-\infty, x_{0}\right)$ of the real line, only. Hence, to guarantee finiteness of the relative entropies $D\left(Z_{n} \| Z\right)$, one has to require that $Z_{n}$ take values in $H$ (which involves a certain requirement on the coefficients $a_{n}$ and $b_{n}$ ). Another important issue is that, as $x \rightarrow x_{0}, \psi(x) \rightarrow 0$ extremely fast, so the finiteness of $D\left(Z_{n} \| Z\right)$ leads as well to an additional strong moment assumption about the distribution of $X_{1}$ near a point. A similar effect may be observed in the case $1 \leq \alpha<2,|\beta|=1$, as well. Here $\psi$ is positive everywhere, but tends to zero extremely fast either near $+\infty$ or $-\infty$ (especially, when $\alpha=1$ ).

Note that the property that $X_{1}$ belongs to the domain of attraction of a stable law of index $0<\alpha<2$ may be expressed explicitly in terms of the distribution function $F(x)=\mathbf{P}\left\{X_{1} \leq x\right\}$. Namely, we have $Z_{n} \Rightarrow Z$ with some $b_{n}>0$ and $a_{n} \in \mathbf{R}$, if and only if

$$
\begin{aligned}
F(x) & =\left(c_{0}+o(1)\right)|x|^{-\alpha} B(|x|) & & (x \rightarrow-\infty), \\
1-F(x) & =\left(c_{1}+o(1)\right) x^{-\alpha} B(x) & & (x \rightarrow+\infty),
\end{aligned}
$$


for some constants $c_{0}, c_{1} \geq 0$ that are not both zero, and where $B(x)$ is a slowly varying function in the sense of Karamata (cf. [I-L], [Z]).

Furthermore, in the non-extremal case the condition $D\left(Z_{n} \| Z\right)<+\infty$ in Theorem 1.1 is equivalent to saying that $Z_{n}$ has a density $p_{n}$ with finite entropy

$$
h\left(Z_{n}\right)=-\int_{-\infty}^{+\infty} p_{n}(x) \log p_{n}(x) d x
$$

(with an additional requirement that $\mathbf{E} X_{1}^{2}<+\infty$, when $Z$ is normal). Once this property is fulfilled for a particular value $n=n_{0}$, it continues to hold for all $n \geq n_{0}$. This will be explained in the next section.

We shall turn to the proof which we divide into several steps. For completeness, the argument will cover the normal case as well.

\section{Remarks on Relative Entropy}

First, let us give necessary and sufficient conditions for the property $D\left(Z_{n} \| Z\right)<+\infty$, especially when $Z$ has a non-extremal stable distribution.

Given a random variable $X$ with density $p$, consider the entropy functional

$$
h(X)=-\int p(x) \log p(x) d x .
$$

In general, it may or may not be defined as a Lebesgue integral. (Here and below, we often omit the limits of integration when integrating over the whole real line.)

It is well-known that, if $X$ has a finite second moment, then $h(X)$ is well-defined, and one has an upper estimate

$$
h(X) \leq h(Z)
$$

where $Z$ is a normal random variable with the same mean and variance as $X$. (Here the value $h(X)=-\infty$ is possible.) This important observation may be generalized with respect to other (not necessarily normal) reference measures.

Proposition 2.1. Let $X$ denote a random variable with density $p$. Assume that $\psi$ denotes a probability density on the real line, such that $\psi(x)=0$ implies $p(x)=0$ a.e., and such that $\mathbf{E} \log ^{+}\left(\frac{1}{\psi(X)}\right)<+\infty$. Then $h(X)$ exists and satisfies

$$
h(X) \leq \mathbf{E} \log \frac{1}{\psi(X)} .
$$

This bound seems to be folklor knowledge and is based on a direct application of Jensen's inequality. To recall the argument, let $Z$ have density $\psi$, and assume that $\psi(x)=0$ implies $p(x)=0$ a.e. The relative entropy given by

$$
D(X \| Z)=\int p(x) \log \frac{p(x)}{\psi(x)} d x=\mathbf{E} \xi \log \xi, \quad \xi=\frac{p}{\psi},
$$

is then well-defined, where the expectation refers to the probability space $(\mathbf{R}, \psi(x) d x)$. Moreover, since $\mathbf{E} \xi=1$, and due to the convexity of the function $t \rightarrow t \log t$, the expectation $\mathbf{E} \xi \log \xi$ exists and is non-negative. But $p \log \frac{p}{\psi}+p \log \frac{1}{p}=p \log \frac{1}{\psi}$ and, by the assumption, 
$\int p \log \frac{1}{\psi} d x$ exists and does not take the value $+\infty$. Hence, $h(X)=\int p \log \frac{1}{p} d x$ also exists and cannot take the value $+\infty$. In addition, the equality

$$
D(X \| Z)=-h(X)+\mathbf{E} \log \frac{1}{\psi(X)}
$$

is justified. Here, the left-hand side is non-negative, so the inequality (2.2) immediately follows.

Choosing for $\psi$ a normal density with the same mean and the same variance as for $X$, one may easily see that (2.2) reduces to (2.1).

As another example, choosing for $\psi$ the density of the Cauchy measure, we obtain a weaker moment condition $\mathbf{E} \log (1+|X|)<+\infty$, which guarantees that the entropy exists and satisfies $h(X)<+\infty$.

Let us return to the definition of the relative entropy,

$$
D(X \| Z)=\int p(x) \log \frac{p(x)}{\psi(x)} d x
$$

assuming that $Z$ has density $\psi$. In order to describe when this distance is finite, one may complement Proposition 2.1 with the following:

Proposition 2.2. Let $X$ be a random variable with density $p$. Assume that

a) $\psi(x)=0 \Rightarrow p(x)=0$ a.e.;

b) $\mathbf{E} \log ^{+}\left(\frac{1}{\psi(X)}\right)<+\infty$;

c) $h(X)$ is finite.

Then $D(X \| Z)$ is finite and is given by (2.3). Conversely, if $\int \psi(x)^{\gamma} d x<+\infty$, for some $0<\gamma<1$, then the conditions $a)-c$ ) are also necessary for the relative entropy to be finite.

Proof. Condition $a$ ) means that the distribution of $X$ is absolutely continuous with respect to the distribution of $Z$ (which is necessary for the finiteness of the relative entropy).

Assuming condition $a$ ), we have $D(X|| Z)<+\infty$, if and only if the integral

$$
I=\int p(x) \log \left(1+\frac{p(x)}{\psi(x)}\right) d x
$$

is finite. Let $I$ indeed be finite. Split the real line into the two sets $A=\left\{x \in \mathbf{R}: p(x) \geq \psi(x)^{\gamma^{\prime}}\right\}$ and $B=\mathbf{R} \backslash A$, where $\gamma<\gamma^{\prime}<1$. Restricting the integration to $A$, we get

$$
I \geq \int_{A} p(x) \log \left(1+\frac{1}{\psi(x)^{1-\gamma^{\prime}}}\right) d x \geq\left(1-\gamma^{\prime}\right) \int_{A} p(x) \log ^{+}\left(\frac{1}{\psi(x)}\right) d x .
$$

Hence, $\int_{A} p(x) \log ^{+}\left(\frac{1}{\psi(x)}\right) d x<+\infty$. On the other hand, using $t^{\gamma^{\prime}} \log (1 / t) \leq C t^{\gamma}(0 \leq t \leq 1)$, we get

$$
\begin{aligned}
\int_{B} p(x) \log ^{+}\left(\frac{1}{\psi(x)}\right) d x & \leq \int_{B} \psi(x)^{\gamma^{\prime}} \log ^{+}\left(\frac{1}{\psi(x)}\right) d x \\
& =\int_{B} \psi(x)^{\gamma^{\prime}} \log \left(\frac{1}{\psi(x)}\right) 1_{\{\psi(x) \leq 1\}} d x \leq C \int_{-\infty}^{+\infty} \psi(x)^{\gamma} d x<+\infty
\end{aligned}
$$


As a result, $\int p(x) \log ^{+}\left(\frac{1}{\psi(x)}\right) d x<+\infty$, that is, $\left.b\right)$ is fulfilled.

But then, by Proposition 2.1, the entropy is well-defined in the Lebesgue sense and, moreover, $h(X)<+\infty$. This justifies writing (2.3), which implies that $h(X)>-\infty$ as well. Hence, the property $c$ ) holds as well.

In the other direction, assuming that $a)-c$ ) are fulfilled, one may start with equality $(2.3)$, which shows that $D(X \| Z)$ is finite.

Thus, Proposition 2.2 is proved.

When $\psi$ is the density of a stable law, the condition $\int \psi(x)^{\gamma} d x<+\infty$ (for some $0<\gamma<1$ ) is fulfilled, so the properties $a)-c$ ) are necessary and sufficient for the finiteness of the relative entropy with respect to $\psi$. In fact, a more detailed conclusion may be stated according to the types of stable laws.

Corollary 2.3. If $Z$ is normal, then $D(X \| Z)<+\infty$, if and only if $X$ has a finite second moment and finite entropy.

Corollary 2.4. If $Z$ has a non-extremal stable distribution, which is not normal, then $D(X|| Z)<+\infty$, if and only if $X$ has a finite logarithmic moment $\mathbf{E} \log (1+|X|)$ and finite entropy.

This follows from Proposition 2.2 and the property (1.1). Let us recall that the condition $\mathbf{E} \log (1+|X|)<+\infty$ insures that the entropy of $X$ exists and, moreover, $h(X)<+\infty$.

The situation where $Z$ has an extremal stable distribution is a bit more delicate, but may be studied on the basis of Proposition 2.2 as well. However, we do not discuss this case here.

These characteristions may be simplified for normalized sums $Z_{n}=\frac{X_{1}+\cdots+X_{n}}{b_{n}}-a_{n}$ with i.i.d. summands as in Theorem 1.1, provided that the sequence $Z_{n}$ is weakly convergent in distribution. Indeed, the property $Z_{n} \Rightarrow Z$, where $Z$ has a stable distribution with parameter $0<\alpha<2$ implies that $\mathbf{E}\left|X_{1}\right|^{s}<+\infty$ for any $0<s<\alpha$, cf. [I-L], [Z]. Hence, $\mathbf{E}\left|Z_{n}\right|^{s}<+\infty$ for all $n \geq 1$, and thus the random variables $Z_{n}$ have finite logarithmic moments.

Corollary 2.5. Assume that $Z_{n}$ converges weakly to a random variable $Z$ with a nonextremal stable limit law, which is not normal. Then, for each $n \geq 1$, the finiteness of the relative entropy $D\left(Z_{n} \| Z\right)$ is equivalent to the finiteness of the entropy of $Z_{n}$.

A similar conclusion holds in the normal case as well, provided that $b_{n} \sim \sqrt{n}$. Here it is well-known that $Z_{n} \Rightarrow Z$ implies that $\mathbf{E}\left|X_{1}\right|^{2}<+\infty$.

Finally, let us mention another property of non-extremal stable distributions.

Corollary 2.6. Assume that $Z$ has a non-extremal stable distribution. If the relative entropy $D\left(Z_{n} \| Z\right)$ is finite for some $n=n_{0}$, it will be finite for all $n \geq n_{0}$.

Proof. By Jensen's inequality, $h(X+Y) \geq h(X)$ for all independent summands such that $h(X)$ exists and $h(X)>-\infty$. If $D\left(Z_{n_{0}} \| Z\right)<+\infty$, then $h\left(Z_{n_{0}}\right)$ is finite according to 
Proposition 2.2. Hence, for the sums $S_{n}=X_{1}+\cdots+X_{n}, h\left(S_{n}\right)$ exists for all $n \geq n_{0}$ and

$$
h\left(S_{n}\right) \geq h\left(S_{n_{0}}\right)=\log b_{n_{0}}+h\left(Z_{n_{0}}\right)>-\infty .
$$

Thus, $h\left(Z_{n}\right)$ exists with $h\left(Z_{n}\right)>-\infty$. In addition, by Corollary 2.4 (if $Z$ is not normal), we also have that $\mathbf{E} \log \left(1+\left|Z_{n_{0}}\right|\right)<+\infty$. By convexity of the function $u \rightarrow \log (1+u)(u \geq 0)$, this yields $\mathbf{E} \log \left(1+\left|X_{1}\right|\right)<+\infty$. In turn, since $\log (1+u+v) \leq \log (1+u)+\log (1+v)$ $(u, v \geq 0)$, we get $\mathbf{E} \log \left(1+\left|S_{n}\right|\right)<+\infty$, for all $n$. In particular, $h\left(S_{n}\right)<+\infty$, according to Proposition 2.1. Therefore, $h\left(Z_{n}\right)$ is finite, and applying Proposition 2.2, we conclude that $D\left(Z_{n} \| Z\right)$ is finite for all $n \geq n_{0}$.

Using Corollary 2.3, a similar argument applies to the normal case as well.

Note that Corollary 2.6 does not extend to the class of extremal stable distributions.

\section{Binomial Decomposition of Convolutions}

Given independent identically distributed random variables $\left(X_{n}\right)_{n \geq 1}$ and numbers $a_{n} \in \mathbf{R}$, $b_{n}>0$, consider the sums

$$
S_{n}=X_{1}+\cdots+X_{n} \quad \text { and } \quad Z_{n}=\frac{S_{n}}{b_{n}}-a_{n} .
$$

If $Z$ is a random variable with an absolutely continuous distribution (not necessarily stable), the condition $D\left(Z_{n} \| Z\right)<+\infty\left(n \geq n_{0}\right)$ used in Theorem 1.1 implies that, for any such $n$, $Z_{n}$ has an absolutely continuous distributions with density, say $p_{n}(x)$. For simplicity, we may and will assume that $n_{0}=1$, that is, already $X_{1}$ has a density $p(x)$. (The case, where $Z_{n}$ have densities starting from $n \geq n_{0}$ with $n_{0}>1$ requires minor modifications only).

Since it is advantageous in the following to work with bounded densities, we slightly modify $p_{n}$ at the expense of a small change in the relative entropy. For a given number $0<b<\frac{1}{2}$, split $H$ into the two Borel sets $H_{1}$ and $H_{0}$, such that $p$ is bounded by a constant $M$ on $H_{1}$ with

$$
b=\int_{H_{0}} p(x) d x .
$$

Consider the decomposition

$$
p(x)=(1-b) \rho_{1}(x)+b \rho_{0}(x),
$$

where $\rho_{1}, \rho_{0}$ are the normalized restrictions of $p$ to the sets $H_{1}$ and $H_{0}$, respectively. Hence, for the convolutions we have a binomial decomposition

$$
p^{* n}=\sum_{k=0}^{n} C_{n}^{k}(1-b)^{k} b^{n-k} \rho_{1}^{* k} * \rho_{0}^{*(n-k)} .
$$

This function represents the density of $S_{n}$.

For $n \geq 2$, we split the above sum into the two parts, so that $p^{* n}=\rho_{n 1}+\rho_{n 0}$ with

$$
\rho_{n 1}=\sum_{k=2}^{n} C_{n}^{k}(1-b)^{k} b^{n-k} \rho_{1}^{* k} * \rho_{0}^{*(n-k)}, \quad \rho_{n 0}=b^{n} \rho_{0}^{* n}+n(1-b) b^{n-1} \rho_{1} * \rho_{0}^{*(n-1)} .
$$


Note that

$$
\varepsilon_{n} \equiv \int \rho_{n 0}(x) d x=b^{n}+n(1-b) b^{n-1}<n b^{n-1}
$$

Finally define

$$
\widetilde{p}_{n}(x)=\frac{b_{n}}{1-\varepsilon_{n}} \rho_{n 1}\left(a_{n}+b_{n} x\right), \quad p_{n 0}(x)=\frac{b_{n}}{\varepsilon_{n}} \rho_{n 0}\left(a_{n}+b_{n} x\right) .
$$

Thus, for the densities $p_{n}$ of $Z_{n}$ we have the decomposition

$$
p_{n}(x)=\left(1-\varepsilon_{n}\right) \widetilde{p}_{n}(x)+\varepsilon_{n} p_{n 0}(x) .
$$

The (probability) densities $\widetilde{p}_{n}$ are bounded and provide a strong approximation for $p_{n}$ regardless of the choice of numbers $a_{n}$ and $b_{n}$ from the definition of $Z_{n}$. In particular, from (3.2) and (3.4) and using $b<\frac{1}{2}$, it follows that

$$
\int\left|\widetilde{p}_{n}(x)-p_{n}(x)\right| d x<2^{-n}
$$

for all $n$ large enough. One of the immediate consequences of this estimate is the bound

$$
\left|\widetilde{f}_{n}(t)-f_{n}(t)\right|<2^{-n} \quad(t \in \mathbf{R})
$$

for the corresponding characteristic functions

$$
\widetilde{f}_{n}(t)=\int e^{i t x} \widetilde{p}_{n}(x) d x, \quad f_{n}(t)=\int e^{i t x} p_{n}(x) d x .
$$

Under mild conditions on $a_{n}$ and $b_{n}$, the approximation (3.5) may be sharpened by using a polynomial weight function in the $L^{1}$-distance.

Lemma 3.1. If $\mathbf{E}\left|X_{1}\right|^{s}<+\infty(s>0)$, and $\left|a_{n}\right|+1 / b_{n}=O\left(n^{\gamma}\right)$ with some $\gamma>0$, then for all $n$ large enough,

$$
\int|x|^{s}\left|\widetilde{p}_{n}(x)-p_{n}(x)\right| d x<2^{-n} .
$$

Proof. We refine arguments from the proof of a similar Lemma 2.1 in [B-C-G]. By (3.4),

$$
\left|\widetilde{p}_{n}(x)-p_{n}(x)\right| \leq \varepsilon_{n}\left(\widetilde{p}_{n}(x)+p_{n 0}(x)\right),
$$

so

$$
\begin{array}{r}
\int|x|^{s}\left|\widetilde{p}_{n}(x)-p_{n}(x)\right| d x \leq \frac{\varepsilon_{n}}{1-\varepsilon_{n}} b_{n}^{-s} \int\left|x-a_{n}\right|^{s} \rho_{n 1}(x) d x \\
+b_{n}^{-s} \int\left|x-a_{n}\right|^{s} \rho_{n 0}(x) d x .
\end{array}
$$

Let $U_{1}, U_{2}, \ldots$ be independent copies of $U$ and $V_{1}, V_{2}, \ldots$ be independent copies of $V$ (that are also independent of all $U_{n}$ ), where $U$ and $V$ are random variables with densities $\rho_{1}$ and $\rho_{0}$, respectively. From (3.2)

$$
\beta_{s} \equiv \mathbf{E}\left|X_{1}\right|^{s}=(1-b) \mathbf{E}|U|^{s}+b \mathbf{E}|V|^{s},
$$

so $\mathbf{E}|U|^{s} \leq \beta_{s} / b$ and $\mathbf{E}|V|^{s} \leq \beta_{s} / b$ (using $b<\frac{1}{2}$ ). Consider the sums

$$
S_{k, n}=U_{1}+\cdots+U_{k}+V_{1}+\cdots+V_{n-k}, \quad 0 \leq k \leq n .
$$


If $s \geq 1$, then by the triangle inequality in the space $L^{s}$ with norm $\|\xi\|_{s}=\left(\mathbf{E}|\xi|^{s}\right)^{1 / s}$, we get

$$
\left\|S_{k, n}\right\|_{s} \leq \sum_{j=1}^{k}\left\|U_{j}\right\|_{s}+\sum_{j=k+1}^{n}\left\|V_{j}\right\|_{s}=k\|U\|_{s}+(n-k)\|V\|_{s} \leq n\left(\beta_{s} / b\right)^{1 / s} .
$$

Hence, $\mathbf{E}\left|S_{k, n}\right|^{s} \leq \frac{\beta_{s}}{b} n^{s}$ and, by Jensen's inequality, $\mathbf{E}\left|S_{k, n}-a_{n}\right|^{s} \leq 2^{s}\left(\frac{\beta_{s}}{b} n^{s}+\left|a_{n}\right|^{s}\right)$.

If $0<s<1$, one can just use

$$
\left|S_{k, n}\right|^{s} \leq\left|U_{1}\right|^{s}+\cdots+\left|U_{k}\right|^{s}+\left|V_{1}\right|^{s}+\cdots+\left|V_{n-k}\right|^{s},
$$

implying that $\mathbf{E}\left|S_{k, n}\right|^{s} \leq n \beta_{s} / b$ and $\mathbf{E}\left|S_{k, n}-a_{n}\right|^{s} \leq n \frac{\beta_{s}}{b}+\left|a_{n}\right|^{s}$. In both cases,

$$
\mathbf{E}\left|S_{k, n}-a_{n}\right|^{s} \leq 2^{s}\left(\frac{\beta_{s}}{b} n^{\max (s, 1)}+\left|a_{n}\right|^{s}\right) .
$$

Hence,

$$
\begin{aligned}
\int\left|x-a_{n}\right|^{s} \rho_{n 1}(x) d x & =\sum_{k=2}^{n} C_{n}^{k}(1-b)^{k} b^{n-k} \mathbf{E}\left|S_{k, n}-a_{n}\right|^{s} \\
& \leq 2^{s}\left(\frac{\beta_{s}}{b} n^{\max (s, 1)}+\left|a_{n}\right|^{s}\right)\left(1-\varepsilon_{n}\right) \\
\int\left|x-a_{n}\right|^{s} \rho_{n 0}(x) d x & =\sum_{k=0}^{1} C_{n}^{k}(1-b)^{k} b^{n-k} \mathbf{E}\left|S_{k, n}-a_{n}\right|^{s} \\
& \leq 2^{s}\left(\frac{\beta_{s}}{b} n^{\max (s, 1)}+\left|a_{n}\right|^{s}\right) \varepsilon_{n} .
\end{aligned}
$$

The two estimates may be used in (3.7), and we get

$$
\int|x|^{s}\left|\widetilde{p}_{n}(x)-p_{n}(x)\right| d x \leq \frac{2^{s+1}}{b_{n}^{s}}\left(\frac{\beta_{s}}{b} n^{\max (s, 1)}+\left|a_{n}\right|^{s}\right) \varepsilon_{n}
$$

It remains to apply (3.2) together with the assumption on $\left(a_{n}, b_{n}\right)$. Lemma 3.1 is proved.

Lemma 3.2. For any $t_{0}>0$, there are positive constants $c$ and $C$ such that, for all $n \geq 2$,

$$
\int_{|t| \geq t_{0} b_{n}}\left|\tilde{f}_{n}(t)\right| d t<C b_{n} e^{-c n}
$$

Proof. Consider the densities $\rho=\rho_{1}^{* k} * \rho_{0}^{*(n-k)}$ appearing in the definition of $\rho_{n 1}$. Their Fourier transforms (i.e., the corresponding characteristic functions) are connected by

$$
\hat{\rho}(t)=\hat{\rho}_{1}(t)^{k} \hat{\rho}_{0}(t)^{n-k} \quad(t \in \mathbf{R}) .
$$

By the Riemann-Lebesgue theorem, $\left|\hat{\rho}_{j}(t)\right| \leq e^{-c}$, for all $|t| \geq t_{0}$ with some constant $c>0$ $(j=0,1)$. Hence, whenever $2 \leq k \leq n$.

$$
|\hat{\rho}(t)| \leq A\left|\hat{\rho}_{1}(t)\right|^{2}, \quad A=e^{-c(n-2)} .
$$


By the decomposition construction, $\rho_{1}(x) \leq M$, for all $x$. Applying Plancherel's formula, we get

$$
\int_{|t| \geq t_{0}}|\hat{\rho}(t)| d t<A \int\left|\hat{\rho}_{1}(t)\right|^{2} d t=2 \pi A \int \rho_{1}(x)^{2} d x \leq 2 \pi A M .
$$

As a consequence, the density $\rho_{n 1}$ satisfies a similar inequality

$$
\int_{|t| \geq t_{0}}\left|\hat{\rho}_{n 1}(t)\right| d t<2 \pi A M\left(1-\varepsilon_{n}\right) .
$$

But, by $(3.3), \widetilde{f}_{n}(t)=\frac{1}{1-\varepsilon_{n}} e^{-i t a_{n} / b_{n}} \hat{\rho}_{n 1}\left(t / b_{n}\right)$, so

$$
\int_{|t| \geq t_{0} b_{n}}\left|\widetilde{f}_{n}(t)\right| d t=\frac{b_{n}}{1-\varepsilon_{n}} \int_{|t| \geq t_{0}}\left|\hat{\rho}_{n 1}(t)\right| d t<2 \pi M b_{n} e^{-c(n-2)} .
$$

Thus, Lemma 3.2 is proved.

Remark 3.3. If $Z_{n} \Rightarrow Z$, where $Z$ has a stable distribution of index $0<\alpha \leq 2$, then necessarily $b_{n} \sim n^{1 / \alpha} B(n)$, as $n \rightarrow \infty$, where $B$ is a slowly varying function in the sense of Karamata (cf. [I-L]). Using standard arguments (cf. e.g. [La]), one can show as well that $a_{n}=o(n)$. Thus, the conditions of Lemma 3.1 for the coefficients $\left(a_{n}, b_{n}\right)$ are fulfilled, once there is a weak convergence.

\section{Entropic Approximation of $p_{n}$ by $\widetilde{p}_{n}$}

We need to extend the assertion of Lemma 3.1 to the relative entropies with respect to the stable laws. Thus, assume that $Z$ has a stable distribution of index $\alpha \in(0,2]$ with density $\psi(x)$ (thus including the normal law). Put

$$
D_{n}=D\left(Z_{n} \| Z\right)=\int p_{n}(x) \log \frac{p_{n}(x)}{\psi(x)} d x, \quad \widetilde{D}_{n}=\int \widetilde{p}_{n}(x) \log \frac{\widetilde{p}_{n}(x)}{\psi(x)} d x,
$$

where $\widetilde{p}_{n}$ are defined according to the decomposition (3.4) for the densities $p_{n}$ of the normalized sums

$$
Z_{n}=\frac{1}{b_{n}}\left(X_{1}+\cdots+X_{n}\right)-a_{n} \quad\left(a_{n} \in \mathbf{R}, b_{n}>0\right) .
$$

Here, as before, $X_{k}$ denote independent identically distributed random variables.

In the lemma below, it does not matter whether or not the sequence $Z_{n}$ converges weakly to $Z$.

Lemma 4.1. Assume that the distribution of $Z$ is non-extremal. If $D_{n}$ is finite for all $n \geq n_{0}$, and $\left|a_{n}\right|+\log b_{n}+1 / b_{n}=O\left(n^{\gamma}\right)$ with some $\gamma>0$, then

$$
\left|\widetilde{D}_{n}-D_{n}\right|<2^{-n}
$$

for all $n$ large enough.

Proof. To simplify the notations (and the argument), we assume that $n_{0}=1$. In particular, $D_{1}=D\left(X_{1} \| Z\right)$ is finite, hence the entropy $h\left(X_{1}\right)$ is finite and $\mathbf{E} \log \frac{1}{\psi\left(X_{1}\right)}<+\infty$, according 
to Proposition 2.2. Define

$$
D_{n 0}=\int p_{n 0}(x) \log \frac{p_{n 0}(x)}{\psi(x)} d x .
$$

By convexity of the function $L(u)=u \log u(u \geq 0)$ it follows that $D_{n} \leq\left(1-\varepsilon_{n}\right) \widetilde{D}_{n}+\varepsilon_{n} D_{n 0}$ and, as easy to see,

$$
D_{n} \geq\left(\left(1-\varepsilon_{n}\right) \widetilde{D}_{n}+\varepsilon_{n} D_{n 0}\right)+\varepsilon_{n} \log \varepsilon_{n}+\left(1-\varepsilon_{n}\right) \log \left(1-\varepsilon_{n}\right) .
$$

By the definition (3.2) of $\varepsilon_{n}$, the two estimates give

$$
\left|\widetilde{D}_{n}-D_{n}\right|<C n\left(n+\widetilde{D}_{n}+D_{n 0}\right) b^{n-1},
$$

which holds for all $n \geq 1$ with some constant $C$. In addition, using an elementary inequality $L((1-b) u+b v) \geq(1-b) L(u)-\frac{1}{e} u-\frac{1}{e}(u, v \geq 0,0 \leq b \leq 1)$, we get from (3.1) that

$$
D\left(X_{1} \| Z\right)=\int L\left(\frac{p(x)}{\psi(x)}\right) \psi(x) d x \geq(1-b) \int \rho_{1}(x) \log \frac{\rho_{1}(x)}{\psi(x)} d x-\frac{2}{e} .
$$

A similar inequality also holds for $\rho_{0}$ with $b$ in place of $1-b$, so

$$
D\left(X_{1} \| Z\right) \geq(1-b) D(U \| Z)-\frac{2}{e}, \quad D\left(X_{1} \| Z\right) \geq b D(V \| Z)-\frac{2}{e},
$$

where $U$ and $V$ have densities $\rho_{1}$ and $\rho_{0}$, respectively. Hence, these random variables have finite entropies, and by Proposition 2.2,

$$
\mathbf{E} \log \frac{1}{\psi(U)}<+\infty, \quad \mathbf{E} \log \frac{1}{\psi(V)}<+\infty
$$

Let $U_{1}, U_{2}, \ldots$ be independent copies of $U$ and let $V_{1}, V_{2}, \ldots$ be independent copies of $V$ (which are independent of all $U_{n}$ as well). Again, by convexity of the function $u \log u$,

$$
\begin{aligned}
\widetilde{D}_{n} & \leq \frac{1}{1-\varepsilon_{n}} \sum_{k=2}^{n} C_{n}^{k}(1-b)^{k} b^{n-k} \int r_{k, n}(x) \log \frac{r_{k, n}(x)}{\psi(x)} d x, \\
D_{n 0} & \leq \frac{1}{\varepsilon_{n}} \sum_{k=0}^{1} C_{n}^{k}(1-b)^{k} b^{n-k} \int r_{k, n}(x) \log \frac{r_{k, n}(x)}{\psi(x)} d x,
\end{aligned}
$$

where $r_{k, n}$ are the densities of the normalized sums

$$
R_{k, n}=\frac{S_{k, n}}{b_{n}}-a_{n}=\frac{1}{b_{n}}\left(U_{1}+\cdots+U_{k}+V_{1}+\cdots+V_{n-k}\right)-a_{n}, \quad 0 \leq k \leq n .
$$

Now, write

$$
D\left(R_{k, n} \| Z\right)=\int r_{k, n}(x) \log \frac{r_{k, n}(x)}{\psi(x)} d x=-h\left(R_{k, n}\right)+\int r_{k, n}(x) \log \frac{1}{\psi(x)} d x,
$$

using the entropy functional $h(R)=-\int r(x) \log r(x) d x$. Adding independent summands to $R$ will only increase the value of this functional. Hence, for any $1 \leq k \leq n$,

$$
\begin{aligned}
h\left(R_{k, n}\right) & =-\log b_{n}+h\left(U_{1}+\cdots+U_{k}+V_{1}+\cdots+V_{n-k}\right) \\
& \geq-\log b_{n}+h(U) .
\end{aligned}
$$


For $k=0$ there are similar relations (with $V$ replacing $U$ ), so whenever $0 \leq k \leq n$, we have $h\left(R_{k, n}\right) \geq-\log b_{n}-C$ with some constant $C$. Inserting in (4.5), we arrive at

$$
D\left(R_{k, n} \| Z\right) \leq \log b_{n}+C+\mathbf{E} \log \frac{1}{\psi\left(R_{k, n}\right)} .
$$

Case 1: $Z \sim N\left(a, \sigma^{2}\right)$ with some $a \in \mathbf{R}$ and $\sigma>0$. Using (4.6) and (3.8)-(3.9) with $s=2$ and the assumption $\mathbf{E} X_{1}^{2}<+\infty$ (due to the assumption $D_{1}<+\infty$, cf. Corollary 2.3), we get

$$
\begin{aligned}
D\left(R_{k, n} \| Z\right) & \leq \log b_{n}+C_{1}+C_{2} \mathbf{E}\left|R_{k, n}\right|^{2} \\
& \leq \log b_{n}+C_{3}+C_{4} \frac{n^{2}}{b_{n}^{2}}+C_{5} a_{n}^{2}
\end{aligned}
$$

with some constants $C_{j}$ depending on $a, \sigma, b$ and $\mathbf{E} X_{1}^{2}$. Using the condition on $a_{n}$ and $b_{n}$, we conclude that $D\left(R_{k, n} \| Z\right) \leq n^{\gamma^{\prime}}$ with some $\gamma^{\prime}$ for all $n$ large enough. Applying this in (4.3)-(4.4), (4.1) yields $\left|\widetilde{D}_{n}-D_{n}\right|=o\left(b_{1}^{n}\right)$, whenever $b<b_{1}<\frac{1}{2}$.

Case 2: $Z$ has a non-extremal stable distribution. By (4.2), we have $\mathbf{E} \log (1+|U|)<+\infty$ and similarly for $V$. In addition, by (1.1),

$$
\mathbf{E} \log \frac{1}{\psi\left(R_{k, n}\right)} \leq A+B \mathbf{E} \log \left(1+\left|R_{k, n}\right|\right)
$$

with some constants $A$ and $B$. To bound the last expectation, one may use the inequality $\log \left(1+\left|u_{1}+u_{2}\right|\right) \leq \log \left(1+\left|u_{1}\right|\right)+\log \left(1+\left|u_{2}\right|\right)$, valid for all real numbers $u_{1}, u_{2}$, together with $\log (1+u) \leq 1 / u+\log u(u>0)$. They yield

$$
\log \left(1+\left|R_{k, n}\right|\right) \leq \log \left(1+\left|a_{n}\right|\right)+\log \left(1+\left|S_{k, n}\right| / b_{n}\right),
$$

while $\log \left(1+\left|S_{k, n}\right| / b_{n}\right) \leq 1 / b_{n}+\log \left(1+\left|S_{k, n}\right|\right)$. From this, we get

$$
\log \left(1+\left|R_{k, n}\right|\right) \leq 1+\log \left(1+\left|a_{n}\right|\right)+\sum_{j=1}^{k} \log \left(1+\left|U_{j}\right|\right)+\sum_{j=k+1}^{n} \log \left(1+\left|V_{j}\right|\right),
$$

and therefore

$$
\begin{aligned}
\mathbf{E} \log \left(1+\left|R_{k, n}\right|\right) & \leq \frac{1}{b_{n}}+\log \left(1+\left|a_{n}\right|\right)+k \mathbf{E} \log (1+|U|)+(n-k) \mathbf{E} \log (1+|V|) \\
& \leq \frac{1}{b_{n}}+\log \left(1+\left|a_{n}\right|\right)+C n
\end{aligned}
$$

Thus, by (4.7) and (4.6), with some constant $C$

$$
D\left(R_{k, n}|| Z\right) \leq C\left(n+\log b_{n}+1 / b_{n}+\log \left(1+\left|a_{n}\right|\right)\right) .
$$

It remains to apply this bound in (4.3)-(4.4), and then (4.1) yields $\left|\widetilde{D}_{n}-D_{n}\right|=o\left(b_{1}^{n}\right)$ with any $b_{1}>b$. One may take $b_{1}=\frac{1}{2}$, and thus Lemma 4.1 is proved. 


\section{Uniform Local Limit Theorem}

Consider the normalized sums $Z_{n}=\frac{1}{b_{n}}\left(X_{1}+\cdots+X_{n}\right)-a_{n}\left(\right.$ where $\left.a_{n} \in \mathbf{R}, b_{n}>0\right)$, associated to independent identically distributed random variables $X_{k}$.

Proposition 5.1. Assume that $Z_{n} \Rightarrow Z$, where $Z$ has a (continuous) density $\psi$. If the random variables $Z_{n}$ have absolutely continuous distributions for $n \geq n_{0}$ with densities, say $p_{n}$, then

$$
\sup _{x}\left|\widetilde{p}_{n}(x)-\psi(x)\right| \rightarrow 0 \quad(n \rightarrow \infty) .
$$

Here $\widetilde{p}_{n}$ denote the modified densities of $p_{n}$, constructed in Section 3 for the case $n_{0}=1$. Necessarily, $Z$ has a stable distribution of some index $\alpha \in(0,2]$, and $b_{n} \sim n^{1 / \alpha} B(n)$, where $B$ is a slowly varying function.

Note that in this proposition it does not matter, whether $Z$ is extremal or not.

Proof. Consider the characteristic functions

$$
\widetilde{f}_{n}(t)=\int_{-\infty}^{+\infty} e^{i t x} \widetilde{p}_{n}(x) d x, \quad f(t)=\int_{-\infty}^{+\infty} e^{i t x} \psi(x) d x
$$

and express the densities via inverse Fourier transforms, while splitting the Fourier integral into the two regions,

$$
\widetilde{p}_{n}(x)-\psi(x)=\frac{1}{2 \pi} \int_{|t| \leq T_{n}} e^{-i t x}\left(\widetilde{f}_{n}(t)-f(t)\right) d t+\frac{1}{2 \pi} \int_{|t|>T_{n}} e^{-i t x}\left(\widetilde{f}_{n}(t)-f(t)\right) d t
$$

with given $t_{0}>0$ and $0<T_{n} \leq t_{0} b_{n}$.

Let $f_{n}$ denote the characteristic functions of $Z_{n}$. By assumption, if $T_{n} \rightarrow+\infty$ sufficiently slowly, then $T_{n} \max _{|t| \leq T_{n}}\left|f_{n}(t)-f(t)\right| \rightarrow 0$. Hence, by (3.6),

$$
T_{n} \max _{|t| \leq T_{n}}\left|\tilde{f}_{n}(t)-f(t)\right| \rightarrow 0 \quad(n \rightarrow \infty),
$$

so that uniformly in all $x$

$$
\widetilde{p}_{n}(x)-\psi(x)=\frac{1}{2 \pi} \int_{|t|>T_{n}} e^{-i t x}\left(\widetilde{f}_{n}(t)-f(t)\right) d t+o(1) .
$$

Moreover, since $f$ is integrable,

$$
\sup _{x}\left|\widetilde{p}_{n}(x)-\psi(x)\right| \leq \frac{1}{2 \pi} \int_{|t|>T_{n}}\left|\widetilde{f}_{n}(t)\right| d t+o(1) .
$$

Recall that the characteristic functions $\widetilde{f}_{n}$ are integrable as well. The integration in (5.2) should also be splitted into the two regions accordingly, and hence the integral itself will be bounded by

$$
\int_{T_{n}<|t|<t_{0} b_{n}}\left|\tilde{f}_{n}(t)\right| d t+\int_{|t|>t_{0} b_{n}}\left|\tilde{f}_{n}(t)\right| d t .
$$


But, by Lemma 3.2, the last integral tends to zero, as long as $b_{n}$ has at most polynomial growth. Using once more (3.6), we arrive at

$$
\sup _{x}\left|\widetilde{p}_{n}(x)-\psi(x)\right| \leq \frac{1}{2 \pi} \int_{T_{n}<|t|<t_{0} b_{n}}\left|f_{n}(t)\right| d t+o(1) .
$$

It remains to apply the following bound derived in [I-L], p.133. There exist constants $c>0$ and $t_{0}>0$, such that $\left|f_{n}(t)\right| \leq e^{-c|t|^{\alpha / 2}}$ for all $n \geq 1$ and $t$ in the interval $|t|<t_{0} b_{n}$. This gives

$$
\int_{T_{n}<|t|<t_{0} b_{n}}\left|f_{n}(t)\right| d t \leq \int_{|t|<t_{0} b_{n}} e^{-c|t|^{\alpha / 2}} d t \rightarrow 0 .
$$

Thus, Proposition 5.1 is proved.

\section{Proof of Theorem 1.1}

Let $X$ and $Z$ be random variables with densities $p$ and $\psi$, such that $\psi(x)=0 \Rightarrow p(x)=0$ a.e. The relative entropy

$$
D(X \| Z)=\int_{-\infty}^{+\infty} \frac{p(x)}{\psi(x)} \log \frac{p(x)}{\psi(x)} \psi(x) d x
$$

is well-defined and may be bounded from above by applying an elementary inequality

$$
t \log t \leq(t-1)+C_{\varepsilon}|t-1|^{1+\varepsilon} \quad(t \geq 0),
$$

where $C_{\varepsilon}$ depends on $\varepsilon \in(0,1]$, only. Namely, it immediately yields

$$
D(X \| Z) \leq C_{\varepsilon} \int_{-\infty}^{+\infty} \frac{|p(x)-\psi(x)|^{1+\varepsilon}}{\psi(x)^{\varepsilon}} d x .
$$

Moreover, letting $\Delta=\sup _{x}|p(x)-\psi(x)|$,

$$
D(X \| Z) \leq C_{\varepsilon} \Delta^{\varepsilon} \int_{-\infty}^{+\infty} \frac{1}{\psi(x)^{\varepsilon}}|p(x)-\psi(x)| d x .
$$

This is a general upper bound which may be used in the proof of Theorem 1.1 in case of a non-normal stable density $\psi$ of index $0<\alpha<2$ and using $X=\widetilde{Z}_{n}$ with modified densities $\widetilde{p}_{n}$. Indeed, by Proposition 5.1,

$$
\Delta_{n}=\sup _{x}\left|\widetilde{p}_{n}(x)-\psi(x)\right| \rightarrow 0 \quad(n \rightarrow \infty) .
$$

In addition, if $Z$ is non-extremal, $\psi$ admits a lower bound $\psi(x) \geq c(1+|x|)^{-(1+\alpha)}$ with some constant $c>0$, cf. (1.1). Hence, by (6.3),

$$
D\left(\widetilde{Z}_{n}|| Z\right) \leq C \Delta_{n}^{\varepsilon} \int_{-\infty}^{+\infty}(1+|x|)^{\varepsilon(1+\alpha)}\left|\widetilde{p}_{n}(x)-\psi(x)\right| d x,
$$

where the constant depends on $\varepsilon$ and $\psi$. But, for an arbitrary $\varepsilon<\frac{\alpha}{1+\alpha}$, so that $s=\varepsilon(1+\alpha)<\alpha$, we see that the last integral does not exceed

$$
\int(1+|x|)^{s}\left|\widetilde{p}_{n}(x)-p_{n}(x)\right| d x+\int(1+|x|)^{s} p_{n}(x) d x+\int(1+|x|)^{s} \psi(x) d x .
$$


All these integrals are bounded by a constant, which follows from Lemma 3.1 and the fact that $\sup _{n} \mathbf{E}\left|Z_{n}\right|^{s}<+\infty$ (which is due to the assumption $Z_{n} \Rightarrow Z$, cf. [I-L]). As a result, we have $D\left(\widetilde{Z}_{n} \| Z\right) \rightarrow 0$, which yields the desired conclusion $D\left(Z_{n} \| Z\right) \rightarrow 0$ in view of Lemma 4.1.

In the normal case $(\alpha=2)$, a similar argument with slight modifications may be applied as well. Without loss of generality, assume that $Z$ is standard normal, i.e., $\psi(x)=\frac{1}{\sqrt{2 \pi}} e^{-x^{2} / 2}$. Now we use (6.2) with $\varepsilon=1$ and $C_{\varepsilon}=1$. More precisely, splitting the integration in (6.1) into the two regions, we get

$$
D(X|| Z) \leq \int_{|t| \leq T} \frac{|p(x)-\psi(x)|^{2}}{\psi(x)} d x+\int_{|t|>T} p(x) \log \frac{p(x)}{\psi(x)} d x
$$

with an arbitrary $T>0$. Furthermore, the first integral on the right-hand side is bounded by

$$
e^{T^{2} / 2} \sqrt{2 \pi} \Delta \int_{|t| \leq T}|p(x)-\psi(x)| d x \leq 2 e^{T^{2} / 2} \sqrt{2 \pi} \Delta .
$$

If additionally $p(x) \leq M,(6.4)$ leads to another general upper bound

$$
D(X|| Z) \leq 2 e^{T^{2} / 2} \sqrt{2 \pi} \Delta+\int_{|t|>T}\left(x^{2}+\log (M \sqrt{2 \pi}) p(x) d x .\right.
$$

Here, again let $X=\widetilde{Z}_{n}$ and $T=T_{n}$. Then the above bound holds with $M=1$ and all $n$ large enough. If $T_{n} \rightarrow+\infty$ sufficiently slow, we have $e^{T_{n}^{2} / 2} \Delta_{n} \rightarrow 0$. On the other hand,

$$
\begin{aligned}
\int_{|t|>T_{n}} x^{2} \widetilde{p}_{n}(x) d x \leq & \int_{-\infty}^{+\infty} x^{2}\left|\widetilde{p}_{n}(x)-p_{n}(x)\right| d x \\
& +\int_{|t|>T_{n}} x^{2} p_{n}(x) d x+\int_{|t|>T_{n}} x^{2} \psi(x) d x .
\end{aligned}
$$

Again, all the integrals tend to zero, in view of Lemma 3.1 and the uniform integrability of the sequence $Z_{n}^{2}$. Hence, by $(6.5), D\left(\widetilde{Z}_{n} \| Z\right) \rightarrow 0$, which, by Lemma 4.1 , proves Theorem 1.1 in the normal case.

\section{REFEREnCES}

[B] A.R. Barron. Entropy and the central limit theorem. Ann. Probab. 14 (1986), no. 1, 336-342.

[B-C-G] S.G. Bobkov, G.P. Chistyakov and F. Götze. Rate of convergence and Edgeworth-type expansion in the entropic central limit theorem. arXiv:1104.3994v1 [math.PR], (2011).

[I-L] I.A. Ibragimov and Ju.V. Linnik. Independent and stationarily connected variables. Izdat. "Nauka", Moscow, 1965, 524 pp.

$[\mathrm{J}] \quad$ O. Johnson. Information theory and the central limit theorem. Imperial College Press, London, 2004 , xiv+209 pp.

[La] J.W. Lamperti. Probability. A survey of the mathematical theory. Second edition. Wiley Series in Probability and Statistics: Probability and Statistics. A Wiley-Interscience Publication. John Wiley \& Sons, Inc., New York, 1996, xii+189 pp. 
[Li] Yu.V. Linnik. An information-theoretic proof of the central limit theorem with the Lindeberg condition. Theory Probab. Appl., 4 (1959), 288-299.

[P] V.V. Petrov. Sums of independent random variables. Springer-Verlag, New York, Heidelberg, Berlin, 1975, x +345 pp.

[Z] V.M. Zolotarev. One-dimensional stable distributions. Probability Theory and Mathematical Statistics, "Nauka", Moscow, 1983, 304 pp.

SERGEy G. Bobkov

School of Mathematics, University of Minnesota

127 Vincent Hall, 206 Church St. S.E., Minneapolis, MN 55455 USA

E-mail address: bobkov@math.umn.edu

Gennadiy P. Chistyakov

FAKultät Für Mathematik, Universität Bielefeld

Postfach 100131, 33501 Bielefeld, Germany

E-mail address: chistyak@math.uni-bielefeld.de

FRIEDRICH GÖTZE

FAKultät Für Mathematik, Universität Bielefeld

Postfach 100131, 33501 Bielefeld, Germany

E-mail address: goetze@mathematik.uni-bielefeld.de 